\title{
A new aspect of neonatal infection to gardnerella vaginalis
}

\begin{abstract}
We report a case of a neonatal infection due to Gardnerella vaginalis in a premature infant born to a dichorionic-diamniotic twin pregnancy. Delivery occurred by caesarean section at 33 weeks of gestational age. Neonatal infection to Gardnerella vaginalis was diagnosed by a positive bacteriological blood culture. Chorioamnionitis and funisitis were confirmed by a placental histological examination. Our observation strongly suggests that Gardnerella vaginalis had crossed intact membranes before delivery. We speculated that this unusual mechanism of contamination might explain the association between bacterial vaginosis and the occurrence of preterm birth with intact membranes.
\end{abstract}

Keywords: gardnerella vaginalis, preterm birth, neonatal infection, chorioamniotitis, funisitis
Volume 6 Issue 3 - 2017

\author{
Dany Al Hamod,' Rami Sabouneh,' Nadine \\ Jawad,' Anne-Elisabeth Mas, ${ }^{2}$ Kim-Da Destot \\ Vong, ${ }^{3}$ David Breich, ${ }^{4}$ Pascal Boileau ${ }^{5}$ \\ 'Neonatal Intensive Care Unit, Department of Pediatrics, Saint \\ George Hospital University Medical Center, Lebanon \\ ${ }^{2}$ Department of Pathology, Univ. Paris-Sud, Antoine Beclere \\ Hospital, France \\ ${ }^{3}$ Neonatal Intensive Care Unit, Department of Pediatrics, Bekaa \\ Hospital, Lebanon \\ ${ }^{4}$ Neonatal Intensive Care Unit, Department of Pediatrics, \\ Centre Hospitalier de Marne-la-Vallee, France \\ Neonatal Intensive Care Unit, Department of Pediatrics, $\mathrm{CHI}$ \\ Poissy Saint-Germain-en-laye, France
}

Correspondence: Dany Hamod, Neonatal Intensive Care Unit, Department of Pediatrics, Saint George Hospital University Medical Center, Beirut, Lebanon. P.O. Box: 166 378, Achrafieh, Beirut II 00 2807, Lebanon, Tel 96।3683209. Email dany_alhamod@hotmail.com

Received: January 27, 2017 | Published: February 28, 2017
Abbreviations: CSF, cerebrospinal fluid; CRP, C-reactive protein;

\section{Introduction}

Gardnerella vaginalis is a Gram negative or variable bacillus commonly present in the normal vaginal flora in 20 to $30 \%$ of pregnant women. It is the most common micro-organism found in bacterial vaginosis which is characterized by a disruption in the balance of vaginal flora with the replacement of lactobacilli by a wide variety of organisms, in particular anaerobic bacteria. ${ }^{1}$ In pregnant women, bacterial vaginosis is associated with an increased risk of intra-amniotic infections $\mathrm{s}^{2,3}$ and with a higher risk of preterm births., ${ }^{4,5}$ Nevertheless, neonatal infections to Gardnerella vaginalis are scarce and the reported cases were considered to be due to an exposure to maternal vaginal flora during delivery ${ }^{6}$ or after a preterm premature rupture of membranes.?

We report a case of neonatal infection to Gardnerella vaginalis in one neonate of a pair of dichorionic-diamniotic twin pregnancy suggesting an ascending pathway of contamination from vagina to the fetus via intact membranes.

\section{Case report}

A dichorionic diamniotic twin pregnancy was obtained by in vitro fertilization, in a 31year old primigravida, with a history of endometriosis and antiphospholipid syndrome, revealed by several thrombo-embolic events on treatment by low-molecular-weight heparin. The pregnancy was complicated by threatened preterm labor at 29 and 32weeks' gestation and was hospitalised for tocolytic (atosiban) and antenatal corticosteroids therapy (betamethasone), until the mother presented in active labor, not responding to tocolytic therapy, febrile $38.1{ }^{\circ} \mathrm{C}$, and no antibiotic therapy despite an elevated CRP of $37 \mathrm{mg} / 1$.
The newborns were delivered by emergency caesarean section for suspected chorioamnionitis at 33 weeks. The first was an $1890 \mathrm{~g}$ female infant, with Apgar scores of 3, 4 and 9 at 1,5 and 10min, respectively. Physical examination at birth revealed a premature infant in mild respiratory distress, prompting continuous positive airway pressure for $1 \mathrm{~h}$.

The second was a male weighing $1780 \mathrm{~g}$, with Apgar scores of 10 at 1,5 and $10 \mathrm{~min}$. Physical examination at birth revealed a premature infant with regular breathing patterns. Cultures from various sites were obtained from both neonates and intravenous ampicillin and gentamicin were administered empirically for $48 \mathrm{~h}$. The neonates remained clinically stable; however, on the fourth day of life the initial blood, gastric, ear and anus swab cultures of the female neonate yielded pure growth of a Gram-negative bacillus identified as Gardnerella vaginalis, whereas those of the male neonate remained negative. Cultures were repeated in addition, a cerebrospinal fluid (CSF) examination was performed. The CSF was normal; however, the serum white blood cell count was $25,900 / \mathrm{mm} 3$ (52\% neutrophils, and $32 \%$ lymphocytes) and a repetitive serum C-reactive protein (CRP) yielded values of 37,40 and $108 \mathrm{mg} / \mathrm{dL}$ at day 1,2 and 4 respectively.

Treatment was modified to intravenous metronidazole $(7.5 \mathrm{mg} /$ $\mathrm{kg} /$ day) for 10days, having a diagnosis of asymptomatic bacteremia to Gardnerella Vaginalis. Follow up of infectious markers decreased at the end of treatment having a CRP value of $10 \mathrm{mg} / \mathrm{l}$. The second neonate's antibiotherapy was stopped after $48 \mathrm{~h}$ of treatment having negative cultures.

Chorioamnionitis was confirmed by placental, membrane and umbilical cord histological examination having a neutrophil polymorph infiltrate into the extraplacental membranes (Figure 1), the wall of the umbilical blood vasculature (vasculitis), and the stroma that surrounds the vessels of the cord (funisitis), (Figure 2). The 
histologic examination of the male neonate's placenta showed a very slight neutrophil infiltration on the placenta's membranes but not in the umbilical cord.

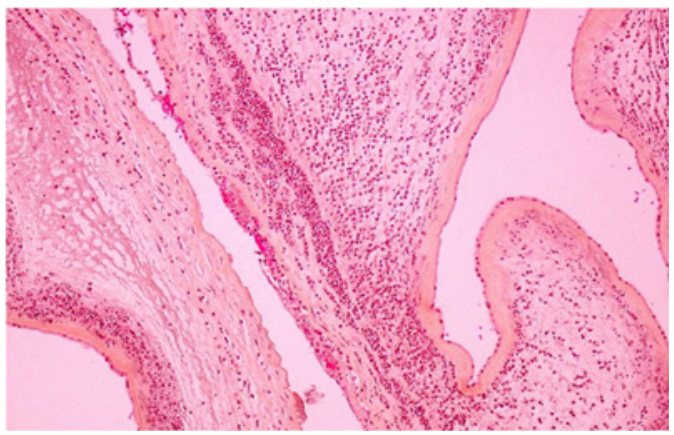

Figure I Chorioamnionitis Acute inflammatory infiltration of neutrophils within the chorionic plate, marginating from the fibrin. HES $\times 4$.

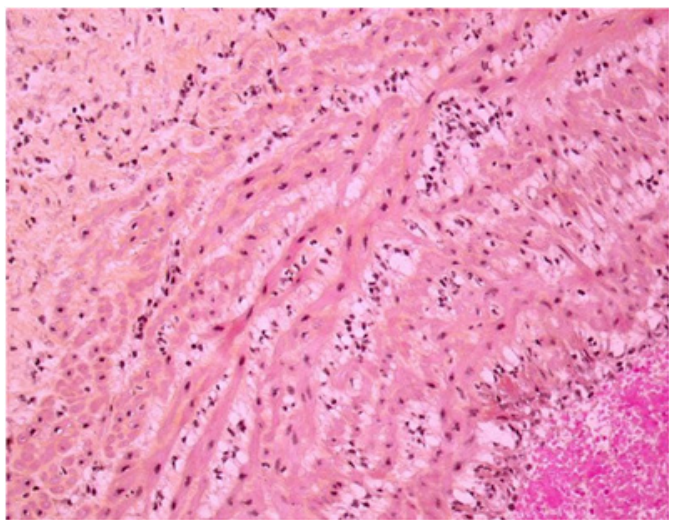

Figure 2 Funisitis

The acute funisitis constitutes the inflammatory foetal response: the foetal inflammatory cells migrate from the umbilical vessels infiltrating the Wharton's jelly. HES $\times 20$.

According to these histological findings of chorioamnionitis in an intact membrane, an antenatal contamination of the female neonate's sac to Gardnerella vaginalis through intact membranes was strongly suspected.

\section{Discussion}

Neonatal infections reported with Gardnerella vaginalis are usually considered to be due to an exposure to maternal vaginal flora during vaginal delivery, ${ }^{6}$ or because of prolonged preterm premature rupture of membrane. ${ }^{7}$ In our case, infants were born by caesarean section with completely intact membranes during the whole pregnancy period. With the aforementioned ways of contamination completely excluded as the sources of neonatal exposure.

The only probable explanation to this single neonatal infection of a dichorionic-diamniotic twin pregnancy could be that the infection to Gardnerella vaginalis occurred by the ascending way from the cervix to the placenta. A second question to raise at this point is why was the female neonate contaminated and not her twin?, and the simple answer was logistic due the close attachment of her placenta to the cervix. This observation aids in reinforcing our hypothesis. The rapid delivery by emergency caesarean section might be the reason why the male neonate was not infected. Keeping in mind that the histology of his placenta showed a very slight neutrophil infiltration but not in the umbilical cord and maybe if delivery was delayed the chance of being infected would have escalated. In addition to the nature of his distal placental insertion from the cervix which may have aided in his exceptional evasion to infection. In conclusion: Gardnerella vaginalis has crossed an intact membrane, generating an inflammatory process leading to chorioamnionitis, vasculitis then funisitis, which probably led to the onset of preterm labor in our case. ${ }^{1,8}$ In 2006, Guaschino et al., ${ }^{3}$ raised the question of the possibility of ascending intrauterine infection, clinical chorioamnionitis, excessive local inflammatory response, clinical symptoms of vaginitis and the risk for preterm birth if the microorganism gained access to the deciduas, in an immunologically hyporesponsive woman who will not be able to control the microbial burden, leaving this hypothesis for further studies. Zabé-Desanges et al., ${ }^{7}$ recently studied the effect of in vitro inoculation of Gardnerella vaginalis to either the amnion or choriodecidua face or to both with measurement of inflammatory markers, this immunological response was previously described by Guaschino et al., ${ }^{3,9}$ This experiment described an inflammatory response in the choriodecidua regardless of the inoculation site. ${ }^{9}$

We speculated that this new way of neonatal infection to Gardnerella vaginalis via an ascending mechanism past intact membranes that might have been weakened by an inflammatory process or an imbalanced immunological response may truly exist. And it may actually strengthen the hypothesis that was published before pending further confirmation.

\section{Acknowledgments}

None.

\section{Conflicts of interest}

Author declares there are no conflicts of interest.

\section{Funding}

None.

\section{References}

1. Pararas MV, Skevaki CL, Kafetzis DA. Preterm birth due to maternal infection: causative pathogens and modes of prevention. Eur J Clin Microbiol Infect Dis. 2006;25(9):562-569.

2. Yudin MH. Bacterial vaginosis in pregnancy: Diagnosis, screening, and managment. Clin Perinatol. 2005;32(3):617-627.

3. Guaschino S, De Seta F, Piccoli M, et al. Aetiology of preterm labour:bacterial vaginosis. BJOG. 2006;113(Suppl 3):46-51.

4. Leitich H, Bodner-Adler B, Brunbauer M, et al. Bacterial vaginosis as a risk factor for preterm delivery: a meta-analysis. Am J Obstet Gynecol. 2003;189(1):139-147.

5. Hillier SL, Nugent RP, Eschenbach DA, et al. Association between bacterial vaginosis and preterm delivery of a low-birth-weight infant. The Vaginal Infections and Prematurity Study Group. $N$ Engl J Med. 1995;333(26):1737-1742.

6. Amaya RA, Al-Dossary F, Demmler GJ. Gardnerella Vaginalis bacteremia in a premature neonate. J Perinatol. 2002;22(7):585-587.

7. Zabé-Desanges C, Rajguru-Kazemi M, Bedu A, et al. [Maternalfetal infection with Gardnerella vaginalis]. Arch Pediatr. 2000; $7(11): 1242-1243$.

8. Newton ER. Preterm labor, preterm premature rupture of membranes and chorioamnionitis. Clin Perinatol. 2005;32(3):571-600.

9. Zaga-Clavellina V, Martha RV-M, Flores-Espinosa P. In vitro secretion profile of pro-inflammatory cytokines IL-1b, TNF-a, IL-6, and of human beta-defensins (HBD)-1, HBD-2, and HBD-3 from human chorioamniotic membranes after selective stimulation with Gardnerella vaginalis. Am J Reprod Immunol . 2012;67(1):34-43. 